\title{
Feedback Control of Boundary Layer Bypass Transition: Experimental and Numerical Progress
}

\author{
Fredrik Lundell* Antonios Monokrousos ${ }^{\dagger}$ \\ Luca Brandt* \\ Linné FLOW Centre, KTH Mechanics, S-100 44 Stockholm, Sweden
}

\begin{abstract}
Experimental and numerical work at the Linné FLOW Centre on active control of transition induced by free-stream turbulence is reviewed and two extensions to previous work are reported. Previously, an experimental setup with upstream sensors and downstream actuators has been built. It has been demonstrated that an ad-hoc control algorithm is able to give a considerable attenuation of the disturbance amplitude downstream of the actuators. Furthermore, large-eddy simulations (LES) of optimal feedback control have been performed for a similar flow configuration and disturbance attenuation as well as transition delay have been obtained. Two extensions are made. First, an effort is made to match the disturbance behavior in the experimental flow case and in the LES. Control is applied in simulations of the matched system aiming at approaching the type of actuation used in the experiments (localized suction). The control law is still computed as optimal feedback of the linear system. As the actuation ability approaches the experiments (where much simpler controllers were used), so does the control effect. Second, system identification (SI) is applied to the experimental data and a more efficient controller is designed. It is made plausible that controllers designed by SI can give considerable improvements in the disturbance attenuation. Implications for future work in the area of active control are discussed.
\end{abstract}

\section{Introduction}

The term boundary layer bypass transition denotes transition scenarios where the dominant instability mechanism is not the exponential growth of two-dimensional Tollmien-Schlichting waves. The most common example is probably transition induced by high levels of free-stream turbulence ${ }^{1}$ (typically above $0.5-1 \%$ of the free-stream velocity). The non-normality of the linearized operator relevant to the dynamics of perturbations of the boundary layer flows, evidenced by the associated non-orthogonal set of eigenmodes, can cause significant transient energy growth. The upstream perturbations which undergo the largest possible growth consist of streamwise counter-rotating vortex pairs. ${ }^{2}$ These vortices lift low-momentum fluid from the wall and push high-momentum fluid from the outer part of the shear layer towards the plate, thus creating elongated regions of alternating accelerated and decelerated fluid, called streaks. The process of lifting of low-momentum fluid from the region near the plate and pulling high-momentum fluid from the outer region is known as lift-up effect. ${ }^{3}$

After their initial generation owing to the lift-up, the streaks grow in strength and become susceptible to high-frequency secondary instabilities. These appear as symmetric and antisymmetric oscillations of the streaks, forming localized regions of chaotic swirly motion, called turbulent spots. ${ }^{1,4,5}$ Subsequently the

\footnotetext{
*Assistant Professor, Linne FLOW Centre, KTH Mechanics, S-100 44 Stockholm, Sweden.

$\dagger$ Graduate student, Linne FLOW Centre, KTH Mechanics, S-100 44 Stockholm, Sweden
} 
(a)

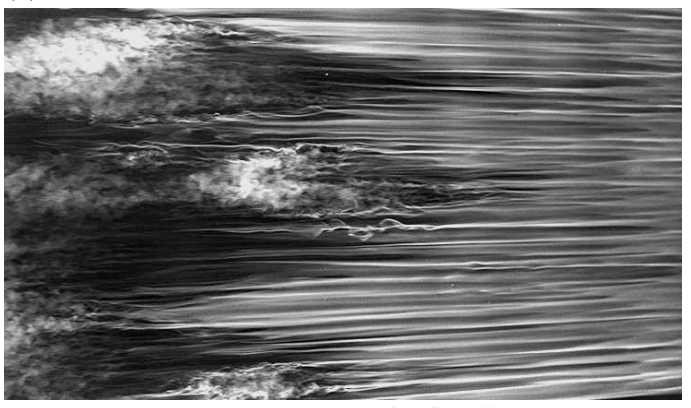

(b)

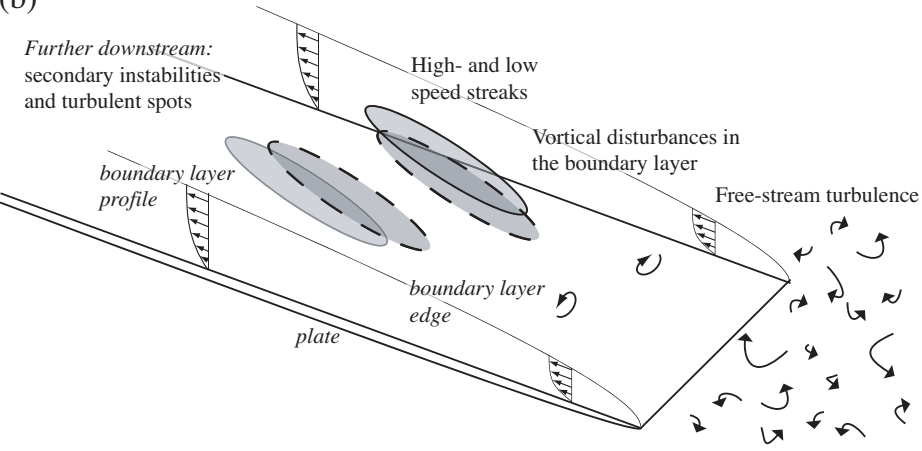

(c)

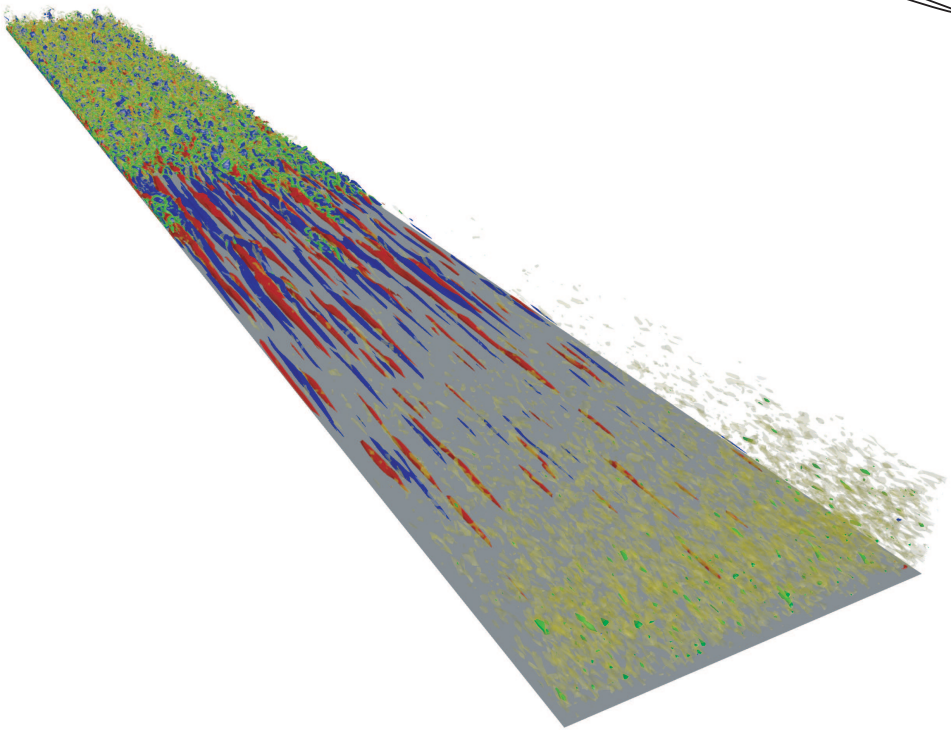

Figure 1. Visualizations (a, c) and schematic (b) of boundary layer transition induced by free-stream turbulence. Flow is from right to left. In the experimental visualization in (a), the smoke is assembled in low velocity regions. In the numerical visualization in (c), the flow is from lower right to upper left. The blue and red isosurfaces correspond to low and high velocity in a box close to the wall shows the flow and the green and yellow isosurfaces indicate the $\lambda_{2}$ vortex identification criterion. The flow is fully turbulent in the second half of the domain. The image in (c) has been provided by Philipp Schlatter (private communication).

spots grow, eventually merge and a fully developed turbulent boundary layer is formed. Visualizations and a schematic of the transition process under free-stream turbulence from experiments and numerical simulations are provided in figure 1. Streamwise streaks can be seen to form upstream inlet, followed by streak oscillations and turbulent spots.

There are several reason for choosing this flow case as a flow-control test-bed. The dynamics governing the disturbance dynamics is well known and fairly complex. As a consequence, the dynamics is known to have similarities with sub-layer streaks in wall-bounded turbulent shear flow. However, the time and length scales in bypass transition are larger and slower, which simplify the experimental realization of the control.

\section{Review of experimental demonstration of feedback control}

An experimental demonstration of feedback control of bypass transition has been reported earlier. ${ }^{6}$ This experiment will now be reviewed in some detail. The data from this experiment will be used in two ways in this paper. One is as the experimental part in a combined numerical/experimental study and the second is as starting point for an analysis in which system identification (SI) is used to predict and indicate possible improvements. 


\section{II.A. Setup}

(a)

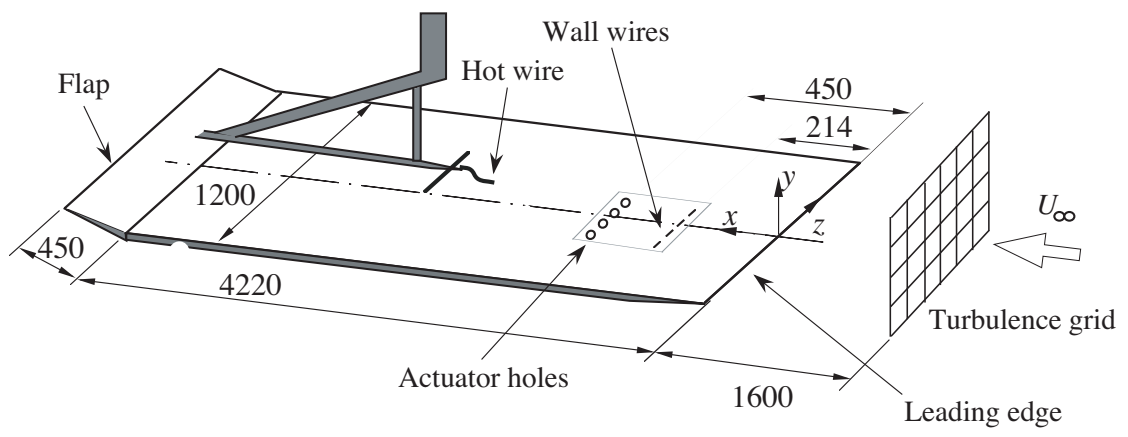

(b)

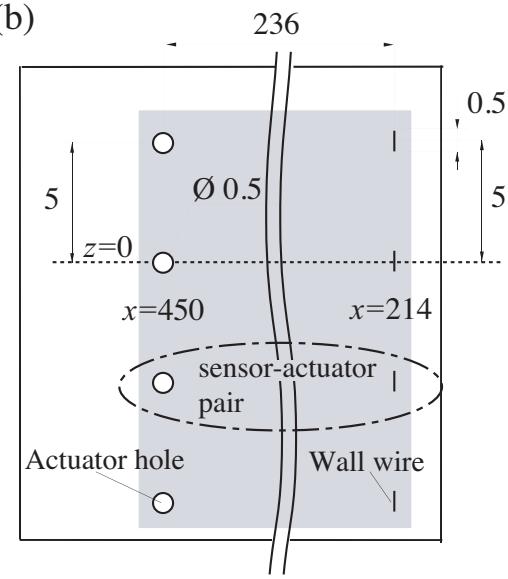

Figure 2. (a) Setup and (b) close up of a control unit. Measures are in mm.

A schematic of the setup is shown in figure 2 (a). The experiments were performed on a flat plate in the MTL wind tunnel at Royal Institute of Technology, Stockholm. Free-stream turbulence was generated by a grid upstream of the plate and the velocity was measured by a hot wire traversed in the flow. The disturbances in the boundary layer over the plate was controlled by one or two control units and one control unit is depicted in figure 2 (b). Two turbulent generating grids are used, one giving a free-stream turbulence level at the leading edge $(T u)$ of $1.4 \%$ and one giving $2.5 \%$. In the first case one control unit is used and in the latter both one and two are used. In the case with more intensive free-stream turbulence, transition occurs within the measured area.

\section{II.A.1. Uncontrolled flow}

The mean velocity and disturbance profiles and streamwise disturbance growth of the uncontrolled flow $(T u=1.4 \%)$ is illustrated with the circles in figure $3(\mathrm{a}-\mathrm{c})$. The mean velocity profiles at different streamwise positions in (a) (marked with the circles) are seen to agree well with the Blasius solution for a laminar zeropressure-gradient boundary layer (shown with the solid line). The disturbance profiles in (b) show the typical disturbance structure in bypass transition: a maximum in the centre of the boundary layer. The disturbances are seen to grow in the streamwise direction in (c).

\section{II.A.2. Control system}

The control units consisted of sensors and actuators. The sensors were wall wires (0.5 mm long) giving a signal indicating the instantaneous local streamwise shear-stress. The actuators were holes $(0.5 \mathrm{~mm}$ diameter) through which suction was turned on and off by fast solenoid valves. The sensors and actuators were connected in pairs so that the suction through an actuator hole was controlled by the sensor straight upstream of it. The control logic (controller) was such that the suction was turned on and off with a delay whenever the streamwise shear (as sensed by the sensor) crossed a threshold. The suction was turned on at instants of low shear stress (indicating the passage of a low speed streak). Each control unit consisted of four neighboring sensor-actuator-pairs and the two units were positioned at $x=214-450 \mathrm{~mm}$ and $x=483-$ $763 \mathrm{~mm}$. The lower value is the position of the sensor and the higher the position of the actuator.

The control system depends on the three variables threshold, delay and suction strength. These parameters were varied during the experiments to obtain a good control effect. Detailed descriptions of the setup and parameter variations are found in the previous publication. ${ }^{6}$ 

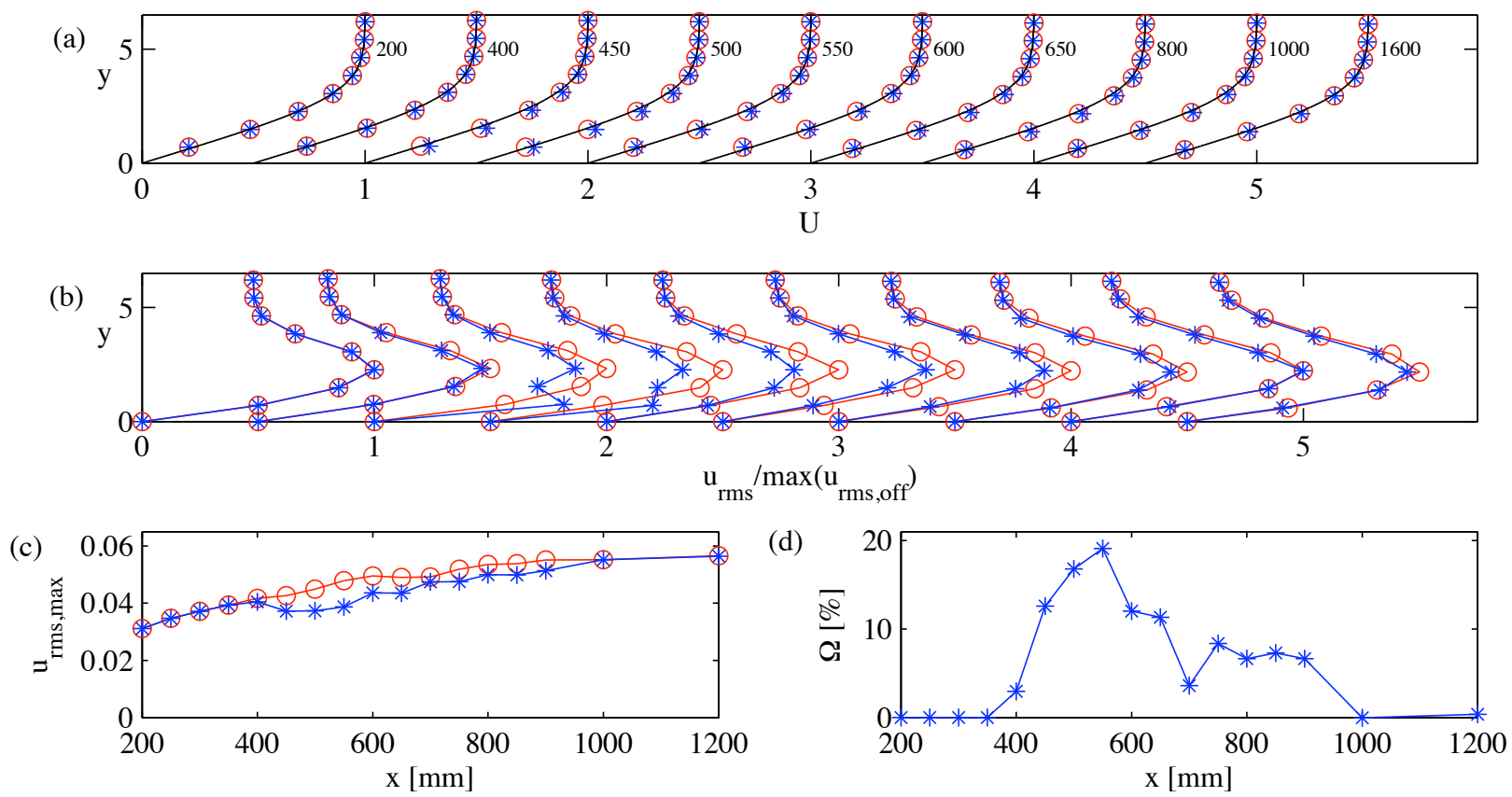

Figure 3. (a) Mean flow, (b) disturbance profiles, (c) disturbance growth and (d) disturbance attenuation in the experiments of Lundell (2007). ${ }^{6}$ Without control (rings, red) and with control (stars, blue), Tu=1.4\%. The line in (a) is the laminar Blasius solution.

\section{II.B. Disturbance attenuation}

The effect of the control is measured by studying the attenuation of the maximum of $u_{r m s}$ at different positions. The disturbance attenuation is quantified as

$$
\Omega_{r m s}=1-\frac{u_{r m s, \max , o n}}{u_{r m s, \max , o f f}}
$$

so that $\Omega_{r m s}$ is the relative decrease of the disturbance level in the boundary layer due to the control.

\section{II.B.1. Case low, $T u=1.4 \%$}

In this case, the control unit at $x=214-450 \mathrm{~mm}$ was used. The results of the control at the low turbulence level are shown in figure 3 (without control with circles and with control with stars). The mean velocity profiles at different streamwise positions in (a) show that there are only minute differences due to the control. Larger effects are seen in the disturbance level (b,c,d). First, in (b), the disturbance level at the center of the boundary layer is seen to decrease with control applied. Over the control hole $(x=450 \mathrm{~mm})$ there is an increase close to the wall due to the control suction. Further downstream, there is a consistent attenuation of the rms-level thanks to the control. This is further demonstrated in (c) where the maximum of $u_{r m s}$ is shown as a function of $x$. In (d), the maximum disturbance attenuation as measured by $\Omega$ is seen to be $20 \%$. Further downstream the attenuation decreases. This is due to new disturbances generated by the free-stream turbulence and disturbances migrating in the spanwise direction.

\section{II.B.2. Case high, Tu=2.5\%}

The disturbance attenuation in the case with high turbulence level are shown in figure 4 . In this case, the turbulence level is high enough to trigger transition within the measured area. Thus, the amplitude development in the streamwise direction (shown in (a)) show three distinct regions: first a linear growth with low amplification (this is the growth of the streaks) followed by a more rapid growth to a maximum 
(a)

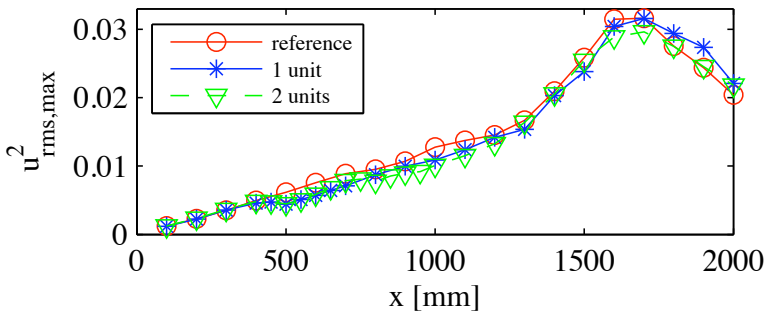

(b)

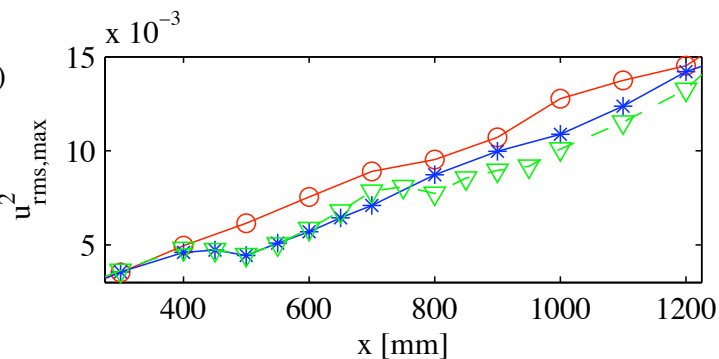

(c)

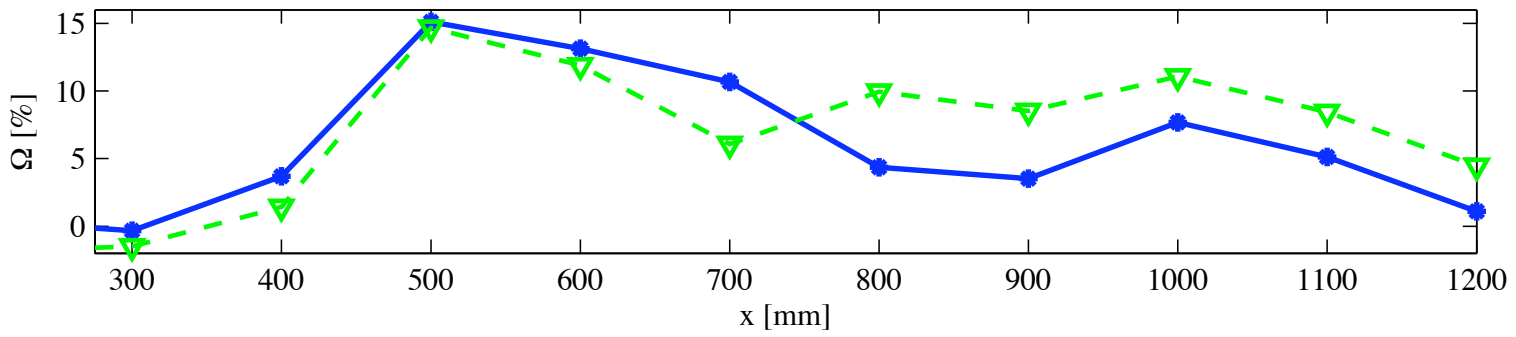

Figure 4. (a,b) Disturbance growth and (c) disturbance attenuation in the experiments of Lundell (2007). ${ }^{6}$ Without control (rings, red), one control unit (stars, blue) and two control units (triangles, green), $T u=2.5 \%$.

(attributed to secondary instabilities and intermittency) and finally a decrease towards the fully developed turbulent case. Both a single (the upstream) and both control units are applied. The control is seen to attenuate the disturbances and the maximum decrease is around $15 \%$. The second control unit does not give a further increase of the disturbance attenuation. Nevertheless, it keeps the downstream attenuation at a higher value than with only the upstream unit.

No transition delay was obtained in these experiment. This was attributed to the fact that the controlled region was very narrow in the spanwise direction. Consequently, the controlled region is contaminated by turbulence from the sides.

\section{Review of numerical simulations of feedback control}

A short description of the previous work on feedback control of bypass transition is reported here. ${ }^{7}$ The aim is to introduce the method and approach pursued. Bypass transition induced by high levels of freestream turbulence above a boundary layer was simulated using direct numerical simulations (DNS) ${ }^{4}$ and large-eddy simulations (LES). A thorough study on different LES models was performed and the ADM-RT subgrid-scale model turned out to be particularly suited for this transitional flow. ${ }^{7}$

\section{III.A. Method}

A linear feedback control scheme was employed in order to reduce the perturbation energy and consequently delay transition. The case of bypass transition represents an extension of the linear approach ${ }^{8}$ to flows characterised by strong nonlinearities. Control was applied by distributed blowing and suction at the wall. Initially, the control signal was based on the full knowledge of the instantaneous velocity field (i.e. full information control). Afterwards in order to relax this unphysical requirement possible only in a numerical simulation, an estimator based on wall measurements was built. This requires the simultaneous simulation of the real flow (plant) and of the estimated flow that is reconstructed from wall measurements only (the two components of the wall shear and the pressure in the present case).

Both the full information controller and the estimator are derived within the Linear Quadratic Gaussian (LQG) framework where a Linear Quadratic Regulator (LQR) is combined with a Kalman filter. ${ }^{9}$ Within this framework a set of linear equations of reduced complexity is used as a model for the physical process to be controlled; with the help of the Lagrange multiplier technique a constrained optimization problem is 

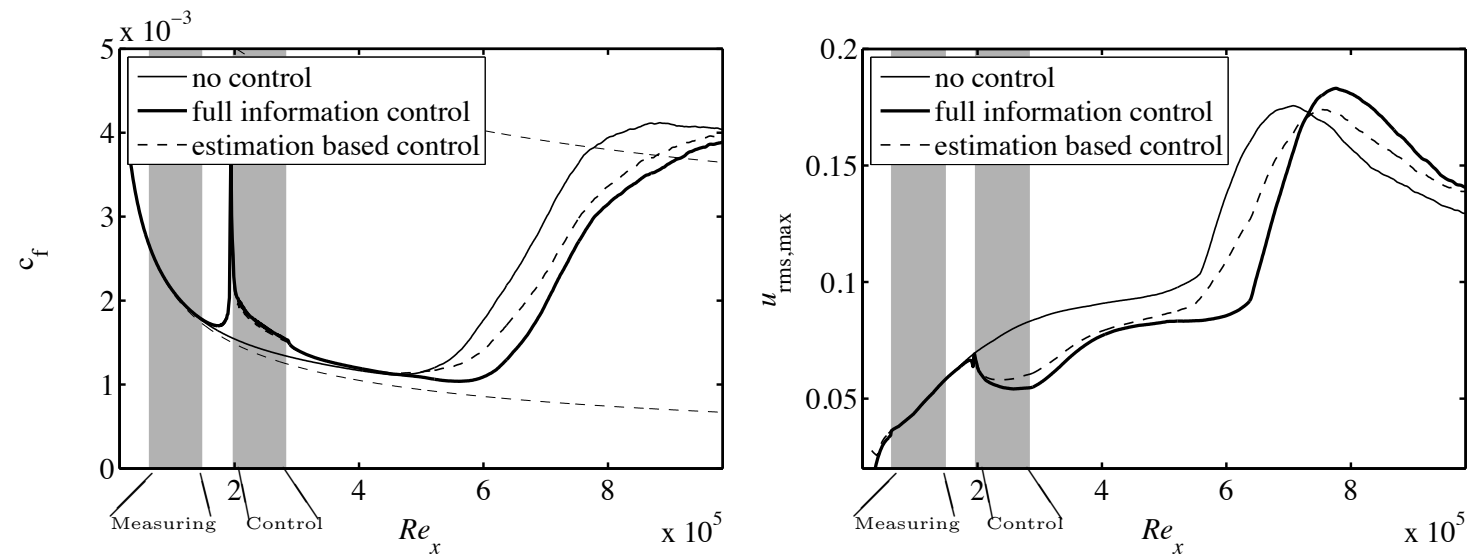

Figure 5. The skin friction coefficient and the wall-normal maximum of the streamwise perturbation velocity are shown for the uncontrolled case and for the simulations with full information control and estimation-based control.

formulated where a chosen quadratic objective function is minimized. The boundary layer flow is modeled by the Orr-Sommerfeld and Squire system governing the evolution of perturbations in parallel flows.

The control and estimation problem can be considered and solved separately. In the full-information control problem the objective function is the kinetic energy of the perturbation velocity. This quantity is minimized while the state of the flow satisfies the governing equation. In the estimation problem a new "state" is considered, the estimated flow, and the objective is to minimize the difference between the wallmeasurements taken from the "real" and the estimated flow; still the perturbations are assumed to satisfy the linearized Navier-Stokes equations. However in the estimation problem a slightly different approach is used. The stochastic framework is employed where the system is assumed to be subject to stochastic excitations. Expected values of the estimation error are therefore examined. ${ }^{10}$ The combination of an estimator and a full information controller is called compensator, where the control law is based on the estimated flow.

\section{III.B. Summary of results}

The results showed that the control was able to delay the growth of the streaks in the region where it is active, which eventually results in a delay of the whole transition process. In figure 5 , the averaged skin friction coefficient and the wall-normal maximum of the streamwise velocity fluctuations are reported to show the transition location and the growth of the streaks inside the boundary layer, respectively. The flow field can be estimated from wall measurements alone: The structures occurring in the "real" flow are reproduced correctly in the region where the measurements are taken. Downstream of this region the estimated field gradually diverges from the "real" flow, revealing the importance of the continuous excitation of the boundary layer by the external stochastic free-stream turbulence. Control based on estimation, termed compensator, was therefore less effective than full information control. Additionally it was found that strong blowing can in fact promote transition due to non-linear effects and thus should be avoided.

\section{Matching of LES and experiments}

In the following we will attempt to apply the control strategy described in the previous section to a numerical simulation that resembles the experimental conditions of the $T u=2.5 \%$ case. Once agreement in the disturbance development has been achieved, we will limit the actuation in the simulation to approach the physical characteristics of the control implemented in the experiment.

For the simulation the code employed ${ }^{11}$ uses Fourier representation in the streamwise and spanwise directions and Chebyshev polynomials in the wall-normal direction. To correctly account for the downstream boundary-layer growth the spatial simulation approach is necessary. This requirement is combined with the periodic streamwise boundary condition by the implementation of a fringe region. ${ }^{12}$ In this region, positioned 
Table 1. Computational box used. Resolution and box dimensions are shown. The box dimensions include the fringe region and are non-dimensionalized with respect to the displacement thickness $\delta_{0}^{*}$ at the inflow $\left(R e_{\delta_{0}^{*}}=300\right)$

\begin{tabular}{cc}
\hline \hline$L_{x} \times L_{y} \times L_{z}$ & $N_{x} \times N_{y} \times N_{z}$ \\
$\delta_{0}^{*}$ & $($ resolution $)$ \\
\hline $2250 \times 60 \times 96$ & $576 \times 121 \times 64$ \\
\hline \hline
\end{tabular}

at the downstream end of the computational box and occupying approximately $10 \%$ of the flow domain, a volume forcing is smoothly raised from zero to force the flow from the outflow to the desired inflow condition. The inflow consists of the laminar Blasius boundary layer with superimposed spatially and temporally varying disturbances mimicking the free-stream turbulence in the present case. ${ }^{4}$

\section{IV.A. Matching of the disturbance growth without control}

The first task is to setup a numerical simulation of the flow that reproduces as close as possible the actual flow of the experiment. However, there are restrictions that make a perfect matching with the experiment virtually impossible. The two main differences are that (i) the code we employ can not include the leading edge and therefore perturbations cannot penetrate directly the boundary layer furthest upstream and (ii) the size of the computational domain is smaller than the wind-tunnel test section and therefore free-stream turbulence of smaller integral length scale can be simulated. The difference in length scales causes different decay rates of the external turbulence and thus different effects upon the underlying boundary layer. Note moreover that in a wider computational domain it would be too expensive to perform all the relevant simulations, especially the extensive parametric study that is performed to match the experimental results and test different control strategies. To facilitate our investigation, it was found that LES can give sufficiently good results. Thus we are aiming at a simulation that reproduces the main features of the conditions in the experiment and not an exact match.

A series of large eddy simulations was carried out to match as close as possible the disturbance growth seen in the experiment. The computational box parameters are reported in table 1 . We vary the turbulence intensity and the integral length scale of the inlet free-stream turbulence and compare the growth of the wall-normal maximum of the streamwise velocity $u_{r m s, \max }$. We tried seven different length scales $l / \delta_{0}^{*}=2.5$, $3.5,4.5,5.5,6.5,7.5,8.5$ and three turbulence intensities at the inlet, $T u=3,3.5,4 \%$.

From figure 6 we see that the case with $T u=3.5 \%$ and $l=4.5 \delta_{0}^{*}$ is closer to the experiment. This is evaluated in terms of initial growth and transition location. The parametric study performed confirms that transition is enhanced when increasing the turbulence intensity and the integral length scale of the turbulence, that is when reducing its decay rate downstream, see also Ref. 7. The turbulence level in the simulation matching the experiment is considerable higher than the one in the experiments. This is due to the fact that there is no leading edge and the integral length scale is shorter due to the limited width of the computational box in the simulations. The shorter length scale results in a faster decay. Consequently, a higher initial turbulence level is needed in the simulations.

\section{IV.B. Optimal control applied to the matched flow}

In the following we will consider as reference uncontrolled case the simulation that was found to agree the most with the experiment (blue solid lines in the coming figures). In this study we are interested on the difference between distributed and localized actuation and in the effect of suction only; we therefore neglect the estimation problem and only consider the full-information control.

First we apply full information control as outlined in section III.A and Ref. 7. This case will provide the control reference case (green solid line in the figures). It can indeed be seen as the best possible performance active control can yield for our configuration. The wall-normal maximum of the $u_{r m s}$ and the attenuation factor $\Omega_{r m s}$ are displayed in figure 7 . It should be mentioned here that the control is applied at the stripe 

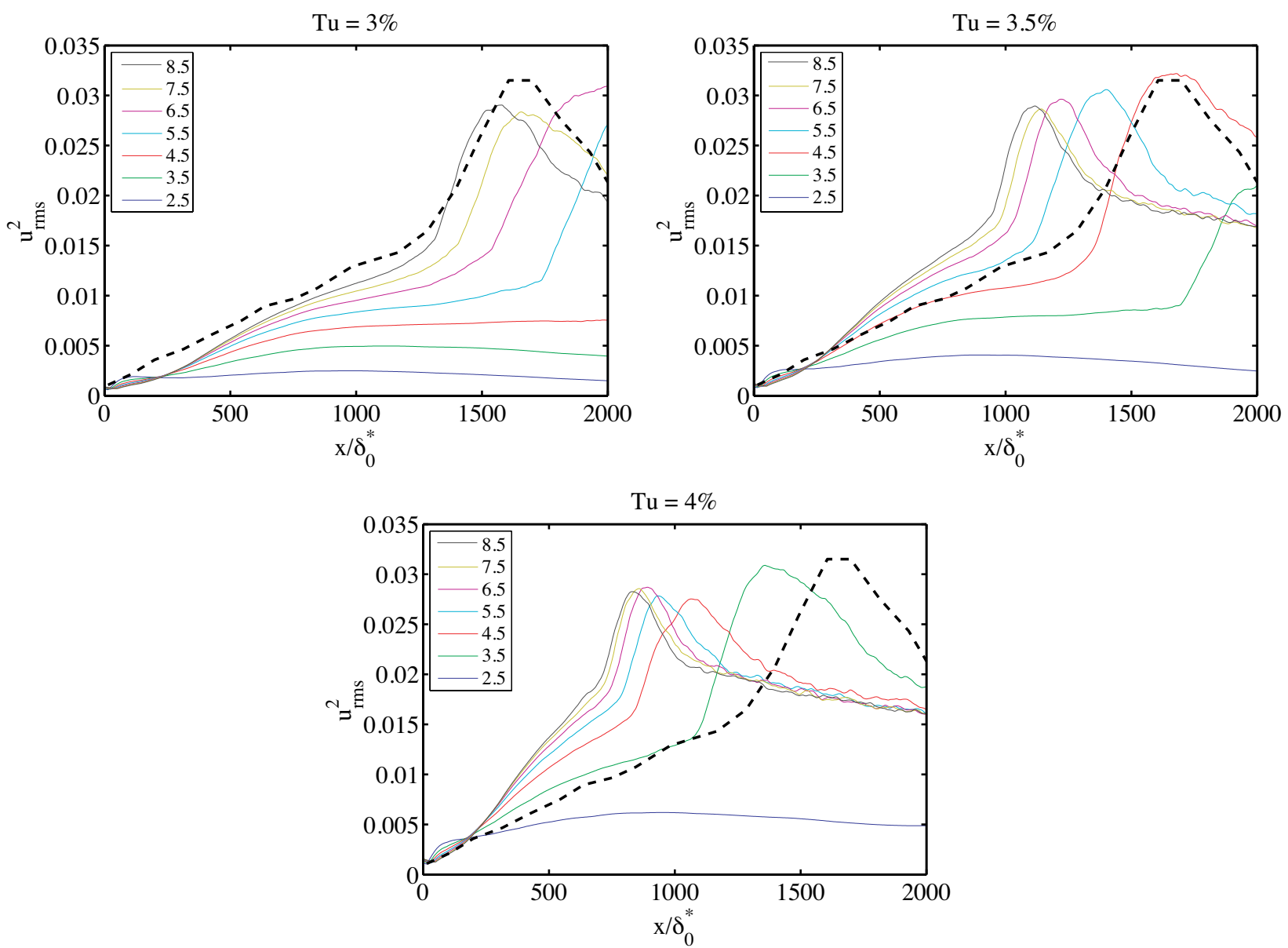

Figure 6. Wall-normal maximum of the streamwise velocity fluctuations $u_{r m s}$. Each plot corresponds to a level of turbulence intensity and each line on the plots to different length scales of the free-stream turbulence at the inlet. The legend shows the length scale in $\delta_{0}^{*}$ units. The dashed black line indicates the experimental data.

starting at $x=350 \delta_{0}^{*}$ and ends at $x=550 \delta_{0}^{*}$. A significant attenuation of the $u_{r m s, \max }$ as well as delay of the transition process can be seen.

At this point it is useful to recall the differences between the actuator in the experiment and in the simulations. These pertains the way the control signal is calculated and the area over which control is applied. In the experiment opposition control is adopted where the amplitude of the suction velocity and the time delay between the sensor an the actuator are varied. In the LES an optimization of the distributed control is performed and no further tuning is required. Note however that the control signal is computed assuming linearly evolving disturbances and parallel base flow. Secondly, it should be mentioned that the control is active over a large area of the plate where relatively weak blowing/suction is applied in the case of the numerical simulations. Conversely, small holes with strong suction velocity are used in the experiment. Further, in the LES we apply control over the full spanwise width of the domain while in the experiment control sets are only stationed near the middle of the plate at an area about $20 \mathrm{~mm}$ wide.

We will now try to wind down these differences. We will not change the control strategy in terms of the way the control signal is calculated but we will focus on the geometrical/functioning aspects of the actuator itself. In that context we will first remove the blowing and keep only the suction. After, we will restrict the area of actuation to spanwise stripes and finally we will also limit the streamwise extension of the area where suction is applied. In the latter last case,we will employ a "cheaper" control in order to result in stronger suction to better mimic the experiment. 

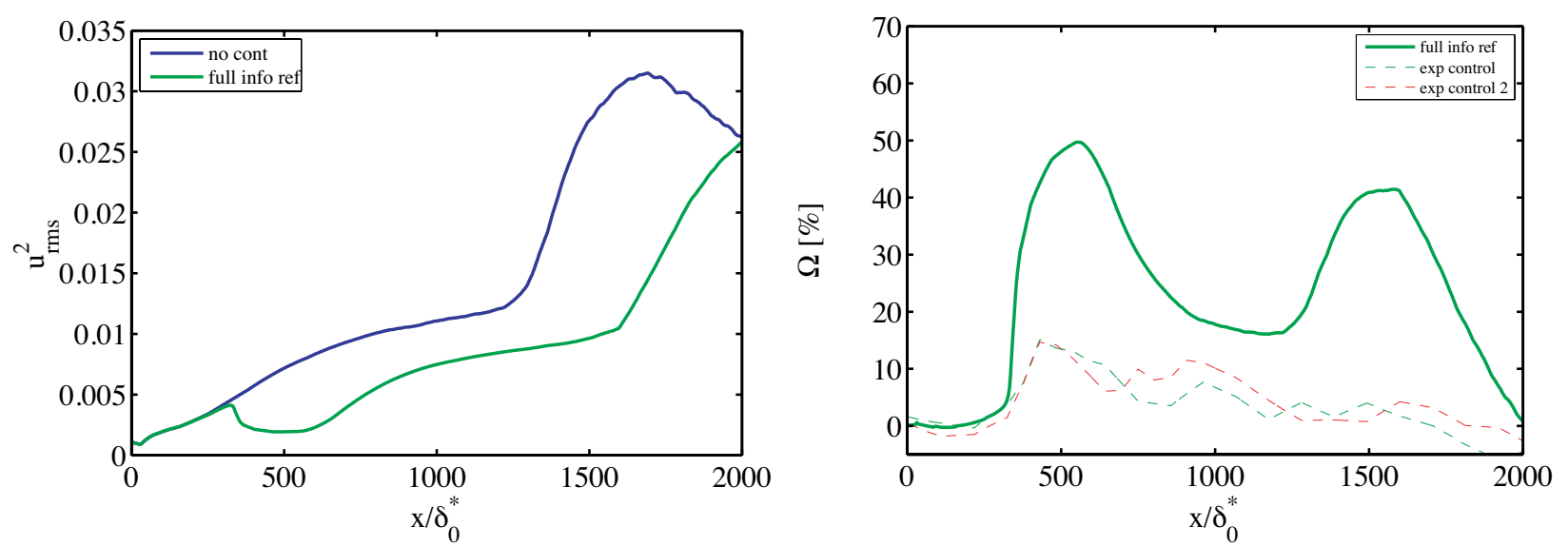

Figure 7. Wall-normal maximum of $u_{r m s}$. Solid lines are simulations. Blue: reference case, Green line: control reference case. Dashed lines are experiments; green line: control with one control set red line: control with two control sets.
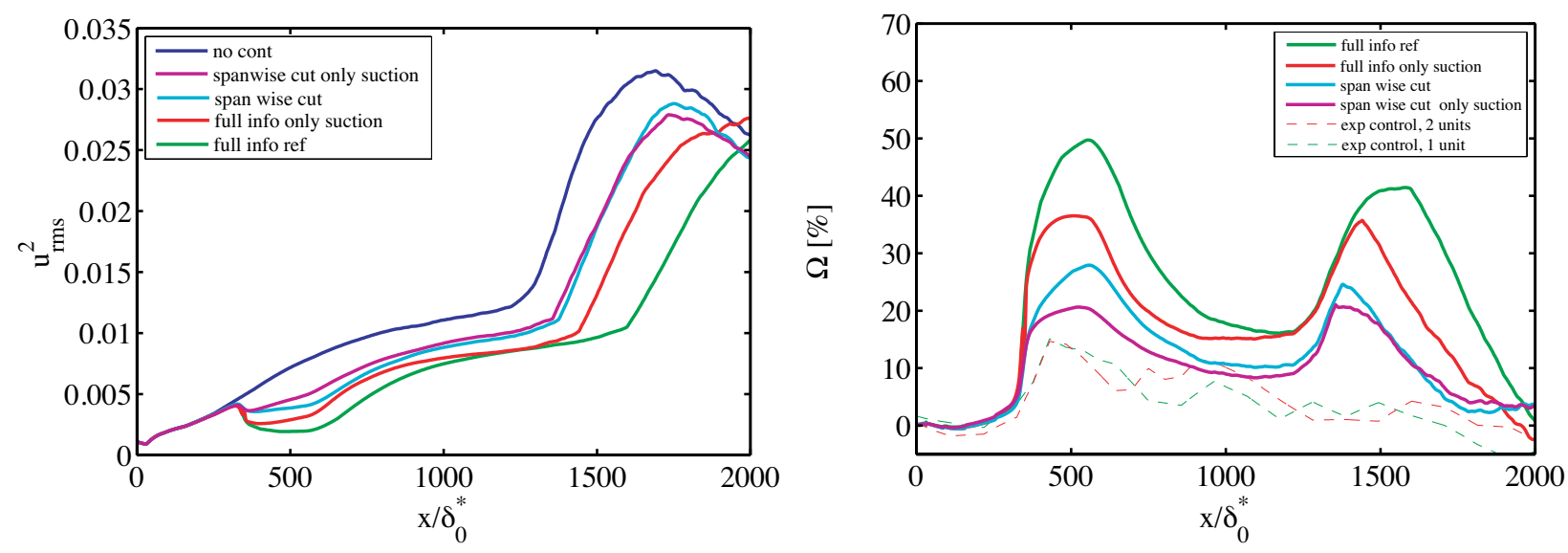

Figure 8. Wall-normal maximum of $u_{r m s}$. Solid lines are simulations: blue: reference case, green line: control reference case, red line: control with only suction case, light blue: control with spanwise cut, purple: control with only suction and spanwise cut. Dashed lines as before.

In figure 8 we see three additional cases where the actuation characteristics are varied. In particular the first case we keep the actuation area the same but cut away all the blowing and keep the suction unchanged (red line in the figure), the second case we keep the blowing and suction unchanged but apply it only in spanwise streaks of width $5 \delta_{0}^{*}$ (light blue line) with a center-to-center distance of $10 \delta_{0}^{*}$ and finally combine the two above cases in both applying only suction and spanwise cutting of the signal (purple line). We see that the performance of the control in the LES is gradually degrading, approaching the experimental results. However the delay in the transition process remains.

Finally in figure 9 we see the results from the simulation where all the previous restriction on the actuator have been applied but also the streamwise extent of the control has been reduced from $200 \delta_{0}^{*}$ to $20 \delta_{0}^{*}$. Additionally we reduce the penalty put on the control during the design process from $l^{2}=10$ to $l^{2}=2$ (see Ref. 7) resulting in stronger suction. In this last case the control effect is almost the same for both the experiment and the simulation near the actuation region but downstream there is a delay of transition only for the numerical control. This can be explained by the fact that in the experiment control is applied near the middle of the plate and where transition occurs fully developed turbulence "invades" the controlled area from the uncontrolled sides. 

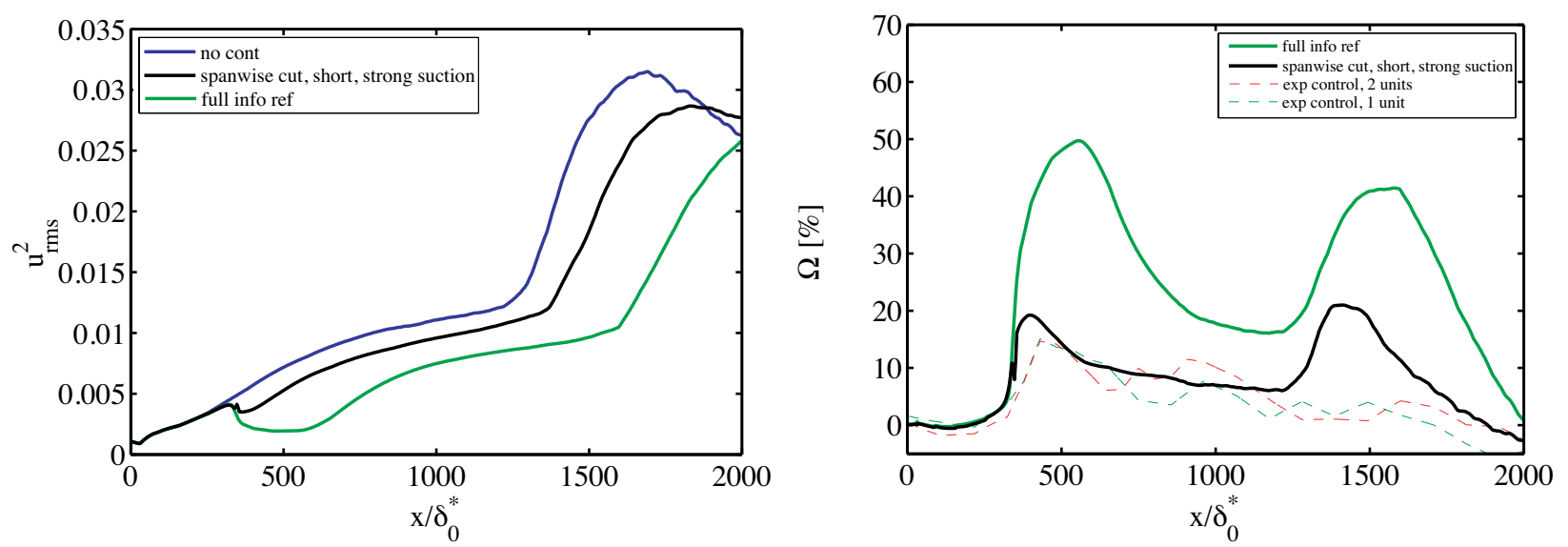

Figure 9. Wall-normal maximum of $u_{r m s}$. Solid lines are simulations: blue: reference case, green line: control reference case, black line: control with only suction, spanwise and streamwise cut and cheaper control (stronger suction). Dashed lines as before.

\section{System identification applied to experimental data}

Linear system identification can be used to model system dynamics for prediction and control purposes. ${ }^{13}$ Here, linear filters will be identified from parts of the experimental time series. Independent data will be used to verify that the filters predict the measured disturbance attenuation. Based on the verified filters, a more efficient control signal is constructed and the estimated disturbance attenuation calculated.

\section{V.A. System identification strategy and verification}

Three signals are used in this work. They are (i) $W(t)$, the fluctuations from the wall-wire sensor, (ii) $U(x, y, t)$, the velocity fluctuations at the position $(x, y, z=0)$ and (iii) $C(t)$, the digital signal controlling the suction at $(x=450 \mathrm{~mm}, z=0)$.

The estimated systems will be used in two ways. The first is to use $W(t)$ and $C(t)$ from experiment and estimate the control effect. This is done to verify the ability of the estimated systems to predict the control result. The second possibility is to use a measured $W(t)$ and design a $C(t)$ which minimizes future flow disturbances. This is done in order to show that considerable improvements might be possible.

The system connecting $W(t)$ and $C(t)$ to the velocity fluctuations is supposed to be:

$$
U(x, y, t)=B_{W}(x, y) W(t)+B_{C}(x, y) C(t)+e(t)
$$

where $e(t)$ is a sequence of random Gaussian noise and $B_{W}$ and $B_{C}$ are polynomials in the shift operator with a delay. With $\Delta t$ as the sampling interval in time the polynomials have the form:

$$
B_{W} W(t)=b_{W}^{1} W\left(t-n_{k W}\right)+b_{W}^{2} W\left(t-\Delta t-n_{k W}\right)+\cdots+b_{W}^{n_{b} W} W\left(t-n_{b W} \Delta t-n_{k W}\right) .
$$

Given the signals from the measurements, standard tools (Ljung 1999) are used to determine the orders $n_{b W}, n_{b C}$, the delays and $n_{k W}, n_{k C}$ and the coefficients $b_{W}^{i}, b_{C}^{i}$.

Since the purpose of these efforts is to improve the disturbance attenuation by the control, the estimation process is verified by its ability to predict the measured disturbance attenuation. The estimated disturbance attenuation is defined as

$$
\Omega_{e s t}(x, y)=1-\left\{B_{W}(x, y) W(t)+B_{C}(x, y) C(t)\right\} / B_{W}(x, y) W(t) .
$$

\section{V.B. Verification of SI results}

The first verification results are shown with stars and squares in figure 10 (a). The stars denote experimental data and the squares the estimated attenuation. The time fraction that the solenoid valves are open is varied. 

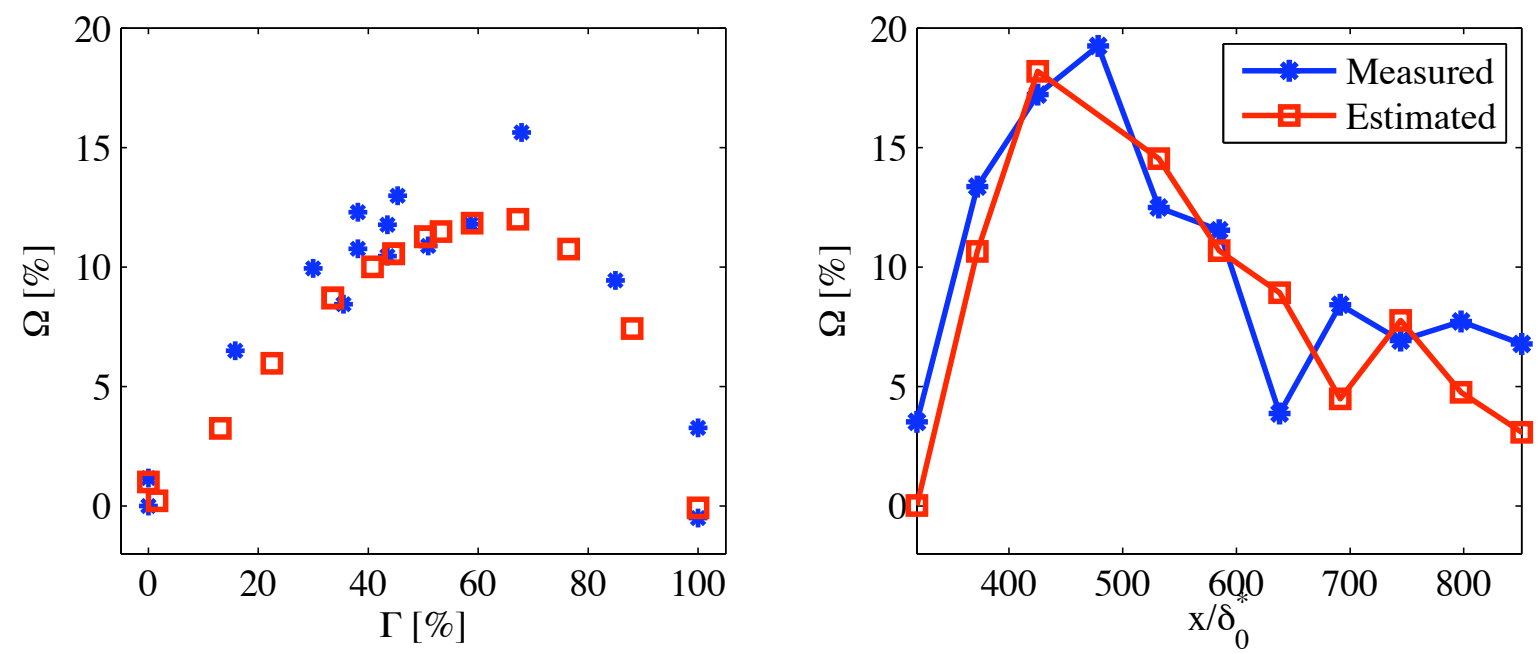

Figure 10. Verification of the ability of the system identification to reproduce the disturbance attenuation. Left: Varying the threshold in the controller, resulting in different $C(t)$ with different $\Gamma$. Note that only one set of filters $B_{W}$ and $B_{C}$ have been used. Right: Measured and estimated disturbance attenuation. For the estimation, one set of $W(t)$ and $C(t)$ (obtained by the threshold-and-delay controller) is used.

This is done by varying the threshold that the wall-shear-stress has to be below for the valve to be open (after a delay). Thus, for each time fraction an individual $W(t)$ and $C(t)$ are used as input for the estimation. The estimation process is seen to reproduce the measurements well.

This ability is further demonstrated in figure 10 (b), where the disturbance attenuation is shown as a function of streamwise position. The reference data without control applied is shown with stars and a dashed line. The stars with the full line show the measured fluctuation level with control applied. Also here, the estimated results are seen to predict the measured data.

\section{V.C. Improved use of control effort}

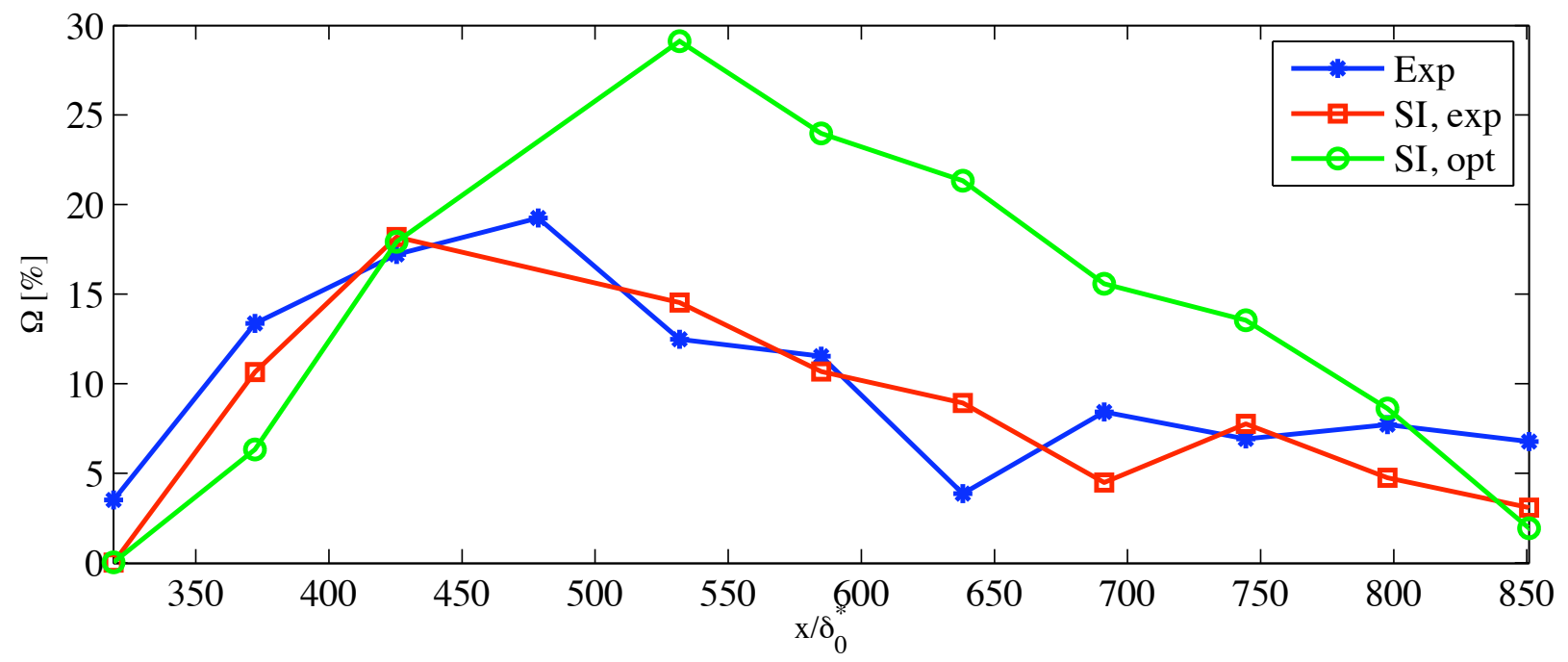

Figure 11. Disturbance attenuation obtained by the optimal $C(t)$.

At this point, it has been demonstrated that the estimated system is able to reproduce the measured 
disturbance attenuation. The system will now be used to predict ultimate control performance. A good sequence $C(t)$ is determined as follows: at each time instant, the disturbance attenuation with and without control applied $(C=1$ and $C=0)$ is estimated. The value of $C$ at this time instant is then chosen as the one which gives the lowest disturbance level. This process is performed using data from the $y$-position of maximum fluctuations and $x / \delta_{0}^{*}=530$. This gives a signal $C(t)$ and the $\Omega_{e s t}$ obtained when using it is shown with circles in figure 11 . From $x / \delta_{0}^{*}=530$ to $x / \delta_{0}^{*}=800$ the disturbance reduction is about twice that obtained in the experiments.

The physical explanation behind these improvements can be understood by studying the step response from actuator to flow. From $x / \delta_{0}^{*}=530$, the step response is non-monotonic and a controller more sophisticated than only linear cancellation (as used in the experiments) is necessary.

\section{Conclusions}

Feedback control of bypass transition has been studied. One experimental ${ }^{6}$ (suction through holes triggered by threshold and delay) and one numerical ${ }^{7}$ (LQR with Kalman filter estimation) study are reviewed. A simulation giving a similar development of the disturbance amplitude as the experiment has been obtained and the LQR has been applied to this simulation. Furthermore, the control effect in one of the experimental cases have been modeled by linear system identification. The main observations are summarized below.

- The LQR with Kalman filter estimation with time and space varying blowing/suction gives much larger initial disturbance attenuation than the experiments (55\% as compared to $15 \%$ ) and a considerable transition delay.

- The initial disturbance attenuation in the simulations approaches the one obtained in the experiments if the capability of the actuator coupled to the LQR is limited towards the ability of the experimental ones (by (i) using only suction, (ii) limiting the actuation to limited spanwise positions and (iii) decreasing the streamwise length of the actuation).

- Compared to the case with complete actuation, a smaller, but still distinct, transition delay is obtained as the actuation ability is decreased.

- The linear system identification (SI) manages to predict the disturbance attenuation well.

- There are indications that SI could be used to improve the disturbance attenuation in future experiments.

Based on these observations, we find it plausible that an experiment in which the full span of the wind tunnel was controlled, would produce a transition delay. Furthermore, we are of the opinion that our result point out durable, flexible, small and cheap actuators as the most important missing technology if feedback control is to be applied to transitional flows for drag reduction. If less advanced actuators have to be used, it might be beneficial to consider their limitations in the controller design.

\section{Acknowledgements}

This work has relied on inspiration and support from professor D. S. Henningson and professor P. H. Alfredsson at KTH Mechanics. Financial support has been received from the Swedish Research Council.

\section{References}

\footnotetext{
${ }^{1}$ Matsubara, M. and Alfredsson, P. H. Disturbance growth in boundary layers subjected to free stream turbulence, J. Fluid. Mech., 2001, 430, 149-168.

2 Andersson, P., Berggren, M. and Henningson, D. S. Optimal Disturbances and Bypass Transition in Boundary Layers, Phys. Fluids, 1999, 11, 134-150.

${ }^{3}$ Landahl, M. T. A note on an algebraic instability of inviscid parallel shear flows, J. Fluid Mech., $1980,98,243-251$.
} 
${ }^{4}$ Brandt, L., Schlatter, P. and Henningson, D. S. Transition in boundary layers subject to free-stream turbulence J. Fluid Mech., 517, 2004, 167-198.

${ }^{5}$ Mans, J., De Lange, H. C. and Van Steenhoven, A. A., Sinuous breakdown in a flat plate boundary layer exposed to free-stream turbulence Phys. Fluids, 2007, 19, 088101.

${ }^{6}$ Lundell F. Reactive control of transition induced by free-stream turbulence: an experimental demonstration, J. Fluid Mech., 2007, 585, 41- 71.

${ }^{7}$ Monokrousos, A., Brandt, L., Schlatter, P. and Henningson, D.S. DNS and LES of estimation and control of transition in boundary layers subject to free-stream turbulence, Int. J. Heat and Fluid Flow, 2008, 29, 841-855.

${ }^{8}$ Chevalier, M., Hoepffner, J., Åkervik E. and Henningson, D. S. Linear feedback control and estimation applied to instabilities in spatially developing boundary layers, J. Fluid Mech., 2007, 588, 163-187.

${ }^{9}$ Friedland, B. Control system design: An introduction to state-space methods, Dover, Mineola, New York, 1986.

${ }^{10}$ Hoepffner, J., Chevalier, M., Bewley, T. R. and Henningson D. S. State estimation in wall-bounded flow systems. Part 1. Perturbed laminar flows, J. Fluid Mech., 2005, 534, 263-294.

${ }^{11}$ Chevalier, M., Schlatter, P., Lundbladh, A. and Henningson, D. S. SIMSON: A Pseudo-Spectral Solver for Incompressible Boundary Layer Flows, KTH Mechanics, Stockholm 2007, Technical Report, KTH/MEK/TR-07/07-SE

${ }^{12}$ Nordström, J., Nordin, N. and Henningson, D. S. The fringe region technique and the Fourier method used in the direct numerical simulation of spatially evolving viscous flows, SIAM J. Sci. Comp., 1999, 20, 1365-1393.

${ }^{13}$ Ljung, L System Identification, theory for the user, Prentice Hall, New York, 1999. 